\title{
MicroscopyEducation
}

\section{Remote Learning Facilitated by MyScope Explore}

\author{
Natalie P. Holmes, ${ }^{1+\star}$ Matthew J. Griffith, ${ }^{2 \dagger}$ Matthew G. Barr, ${ }^{3}$ \\ Nicolas C. Nicolaidis, ${ }^{3}$ Vijay Bhatia, ${ }^{1}$ Michael Duncan, ${ }^{4}$ \\ Ingrid McCarroll, ${ }^{1}$ Jenny Whiting, ${ }^{5}$ Paul C. Dastoor, ${ }^{3}$ and \\ Julie M. Cairney ${ }^{1,2,5}$
}

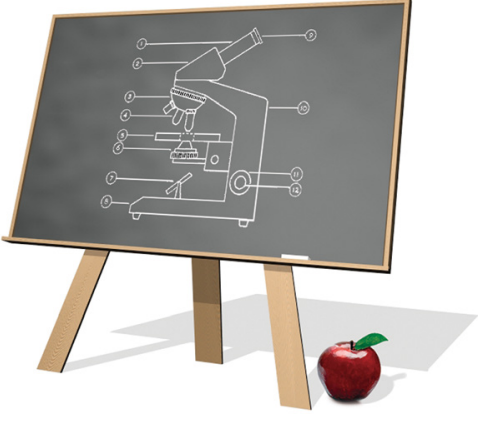

${ }^{1}$ Australian Centre for Microscopy and Microanalysis, The University of Sydney, Sydney, NSW 2006, Australia

${ }^{2}$ School of Aerospace, Mechanical and Mechatronic Engineering, The University of Sydney, Sydney, NSW 2006, Australia

${ }^{3}$ Centre for Organic Electronics (COE), University of Newcastle, Callaghan, NSW 2308, Australia

${ }^{4}$ Hawkesbury Institute for the Environment, Western Sydney University, Penrith, NSW 2751, Australia

${ }^{5}$ Microscopy Australia Headquarters, The University of Sydney, Sydney, NSW 2006, Australia

†These authors contributed equally.

*natalie.holmes@sydney.edu.au

\begin{abstract}
In response to the requirements imposed by the COVID-19 pandemic in 2020 , we developed a remote learning undergraduate workshop for 44 students at the University of Newcastle by embedding scanning electron microscope (SEM) images of Maratus (Peacock) spiders into the MyScope Explore environment. The workshop session had two main components: 1) to use the online MyScope Explore tool to virtually image scales with structural color and pigmented color on Maratus spiders; 2) to join a live SEM session via Zoom to image an actual Maratus spider. In previous years, the undergraduate university students attending this annual workshop would enter the Microscopy Facility at the University of Newcastle to image specimens with SEM; however, in 2020 the Microscopy Facility was closed to student visitors, and this virtual activity was developed in order to proceed with the educational event. The program was highly successful and constitutes a platform that can be used in the future by universities for teaching microscopy remotely.
\end{abstract}

Keywords: microscopy, remote learning, virtual tools, outreach, structural color

\section{Introduction}

In this article we detail the virtual lab that was developed, including both the MyScope Explore simulation and the remote interactive live SEM demonstration, showcasing the journey to expanding remote learning tools during the COVID-19 pandemic. In 2011 Microscopy Australia (formerly AMMRF) created-and now maintains-the online MyScope microscopy simulation training platform, which is a highly accessed tool. In the period from June 25, 2020 to June 24, 2021, MyScope was visited by 141,699 users and received 1,182,252 page views. The user visits were from a total of 184 countries, with the top 7 countries accessing the site comprising $57 \%$ of all users, including the USA, India, UK, Australia, Pakistan, Germany, and China.

The MyScope (myscope.training) online learning modules, including MyScope Explore (myscope-explore.org.au), were developed to provide an online learning environment for those who want to learn about microscopy [1-3]. The platform provides insights into the fundamental science behind different types of microscopies, explores what can and cannot be measured by different material systems, and provides a realistic simulated operating experience for state-of-the-art microscopes (Figure 1).

\section{The Workshop Platform - Pre-COVID-19 versus Now}

The University of Newcastle Centre for Organic Electronics (COE) Spring and Winter Schools were developed as a platform for teaching undergraduate students from a range of disciplines and for developing and testing laboratory exercises that can be used beyond this event at other teaching institutions [4]. The electron microscopy component of the 3-day workshop is usually comprised of 45-minute excursions to the electron microscopy and X-ray (EMX) facility, whereby the undergraduate students participate in an electron microscopy demonstration of all stages of data collection, including sample loading, alignment, and detector selection-to careful selection of aperture size, working distance, brightness, contrast, focus, and magnification settings for image optimization. For the 2020 workshop, held October 7-9, undergraduate students were not permitted to enter the EMX facility due to capacity limits associated with social distancing rules imposed during the COVID-19 pandemic; hence, a remote learning program was developed. The remote learning program comprised two main components:

1. MyScope Explore Online Simulation: A remote learning simulation environment where the students simulated measuring Maratus (Peacock) spider specimens.

2. Interactive Live SEM Session: A practical session where the students remotely measured the real Peacock spider specimens on a Zeiss Sigma VP field emission SEM at the University of Newcastle EMX facility by interacting over Zoom with a scientist operating the microscope (Figure 2). 


\section{First-and-Only AFM-Based Viscoelastic Technique that Ties Directly to Bulk DMA}
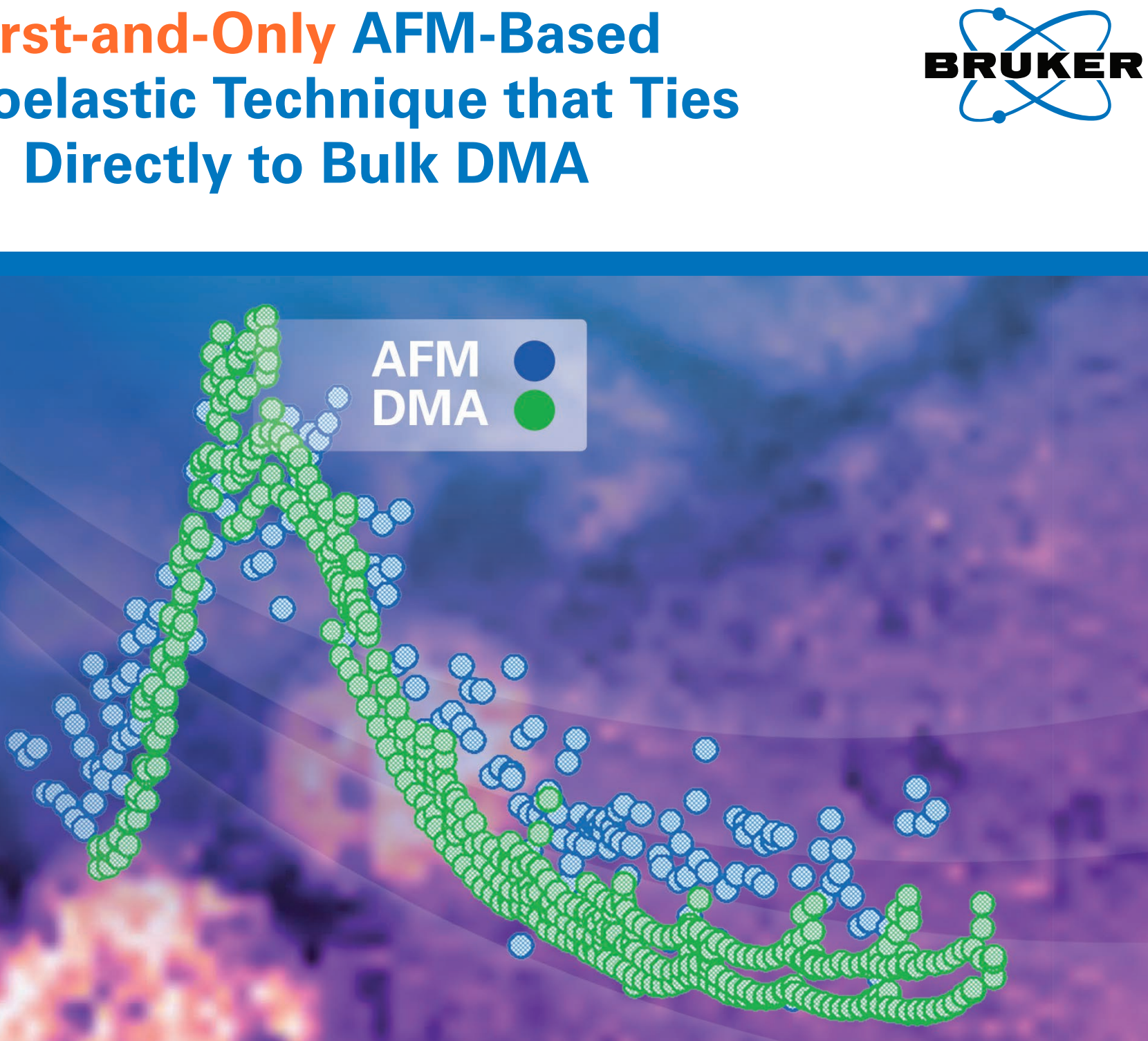

\section{AFM-nDMA mode - Addressing Nanoscale Domains with Highest Resolution}

How do nanoscale phases differ from bulk? What are viscoelastic properties right near an interface or in an interphase? How does this affect load transfer, as a function of temperature? Bruker's new AFM-nDMA mode allows researchers to answer these types of questions. For the first time an AFM can provide complete and quantitative viscoelastic analysis of polymers at the nanoscale, probing materials at rheologically relevant frequencies, in the linear regime. Proprietary dual-channel detection, phase-drift correction, and reference frequency tracking enable a small strain measurement in the rheologically relevant $0.1 \mathrm{~Hz}$ to $20 \mathrm{kHz}$ range for nanoscale measurements of storage modulus, loss modulus, and loss tangent that tie directly to bulk DMA.

Only Bruker's AFM-nDMA mode

- Provides accurate moduli and loss tangent at bulk DMA frequencies - a first for AFM

- Delivers absolute calibration with quantified load and adhesion - and no need for a reference sample
- Creates master curves with WLF and Arrhenius analyses from AFM data

- Discovers nanomechanical effects that conventional AFM approaches might miss

Your research deserves the very best that AFM technology has to offer!

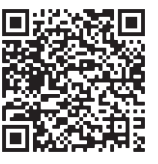

Visit www.bruker.com/AFM-nDMA, email productinfo@bruker.com or call $+1.805 .967-2700$ for more information. 


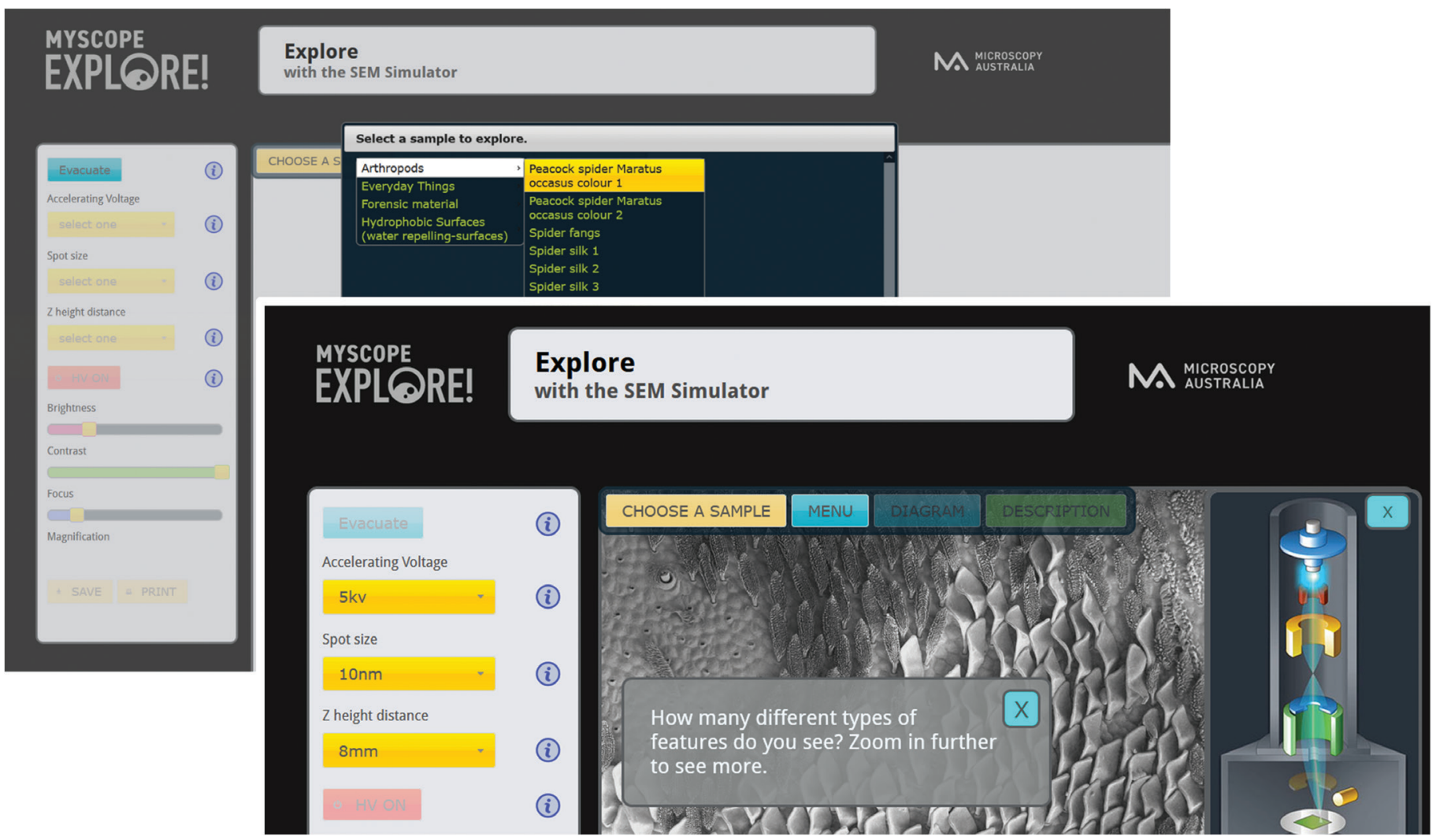

Figure 1: Images of the MyScope Explore SEM simulation environment, which can be accessed at myscope-explore.org.au/virtualSEM_explore.html.
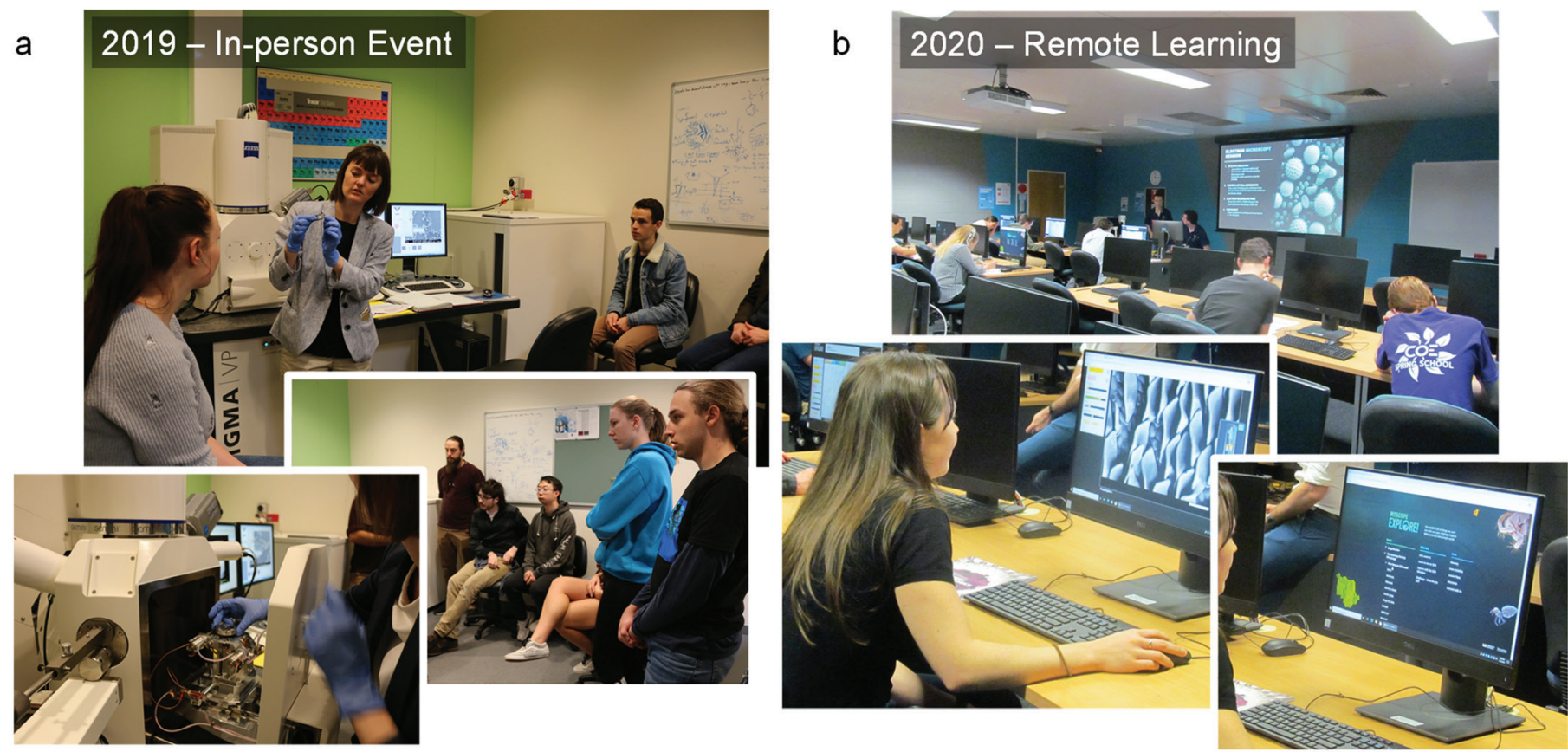

Figure 2: (a) Teaching SEM at the University of Newcastle workshop in 2019, an in-person event. (b) Teaching SEM for the same workshop in 2020 via a remote learning program during the COVID-19 pandemic, comprising both an online MyScope Explore simulation component and an interactive live SEM session via Zoom. In 2020, social distancing rules were in place, a major driver for the development of the remote learning program. Note that in the future, students will also be able to join from home, rather than a university computer lab space as pictured. 


\section{The Undergraduate Student Cohort}

The undergraduate student cohort in 2020 comprised 44 students from a range of degrees, including Bachelors of Science, Engineering, Mathematics, Education, Computer Science, Biomedical Science, and Technology. Students ranged from first year to fourth (final) year undergraduate levels, and all were enrolled at the University of Newcastle.

\section{A Unique Specimen for Teaching - Structural Color in Maratus (Peacock) Spiders}

Maratus occasus is a recently discovered Peacock spider species in Queensland, Australia, belonging to the Maratus tasmanicus group (Figures 3a and 3b) [5]. This particular specimen was chosen for the workshop to complement the samples examined in previous years when students had studied organic semiconductor nanoparticle films (at Spring and Winter Schools) and self-assembled colloidal nanoparticle photonic crystals (in the PHYS3390 course). The choice of naturally occurring structural-colored specimens in 2020 enabled us to build upon previous years' work imaging nanostructured materials that interact with light.

Structural color occurs in nature in many organisms, including butterflies [6], peacocks [7], spiders [8,9], and rainforest beetles [10]. In addition, structural color occurs in naturally occurring minerals such as opal gemstones and can also be produced synthetically, for example, in self-assembled colloidal nanoparticle arrays [11] and 3D printed microscale objects [12]. Structural color results from the interaction of light waves with a structural feature that exhibits the same order of size as the wavelength of light, noting the wavelength of visible light ranges from 380 to $750 \mathrm{~nm}$. Spiders employ a variety of structural coloration mechanisms, including multilayer reflectors, three-dimensional photonic crystals, and diffraction gratings.

Structural color in Australian Peacock spiders is varied, from blue in the scales of Maratus occasus and Maratus splendens $[5,13]$ to super-black in Maratus speciosus and Maratus karrie [14] to full-spectrum rainbow iridescence in Maratus
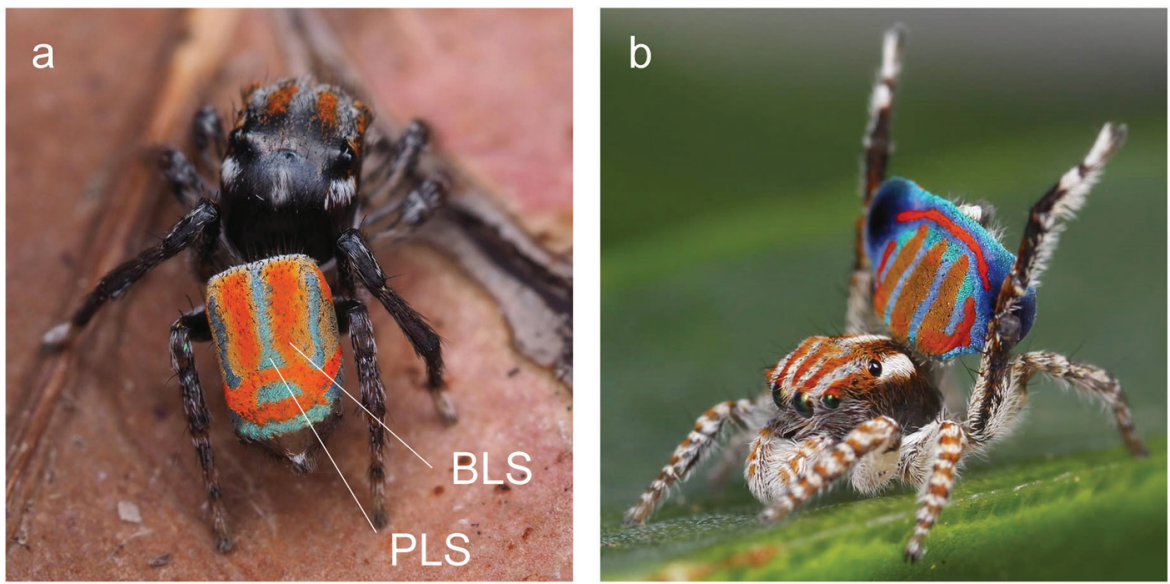

Figure 3: Maratus spiders containing structurally colored blue scales located on the abdomen (dorsa opisthomal plate). (a) Photograph of species Maratus occasus (male) collected from Lake Broadwater, Queensland. (b) Photograph of species Maratus tasmanicus (male) collected from Point Cook, Victoria. The typical species size for Maratus occasus and Maratus tasmanicus is $4 \mathrm{~mm}$. In (a) the blue plate-like scales (PLS) (Type I) and orange brush like-scales (BLS) (Type II) are annotated. Photographs courtesy of Joseph Schubert. robinsoni and Maratus chrysomelas [9]. The super-black regions in the species Maratus speciosus and Maratus karrie reflect as little as $0.44 \%$ and $0.35 \%$ of visible light, respectively, owing to their nanoscale structures. Both species evolved microlens arrays, comprising tall and tightly packed cuticular bumps. The super-black is a combination of pigment and structural effects. The microlens arrays achieve structurally assisted enhanced absorption of light by melanin pigment. The species Maratus robinsoni displays angle-dependent rainbow iridescence attributed to their scales that are comprised of $2 \mathrm{D}$ nanogratings on microscale $3 \mathrm{D}$ convex surfaces.

Maratus occasus is a newly discovered species, hence, a detailed investigation of the structural and pigmented color origins of its scales does not exist in the literature. Therefore, we draw on the literature from similar species in the spider genus Maratus, such as Maratus splendens, with scales of similar color that have been studied in detail [13]. Maratus occasus has two types of scales, which the students investigated during the workshop. Type I: plate-like scales, which are blue in color, and Type II: brush-like scales, which are orange in color (Figure 3a, Figure 4). The brush-like scales, being similar in structure to the red brushlike scales of Maratus splendens, are likely to have pigments that give rise to their color. The plate-like scales, being similar in structure to the blue plate-like scales of Maratus splendens, are likely to have structural color origins. The dual thin film structure of the chitin plate-like scales, with an internal filament array (Figure 4b), likely leads to the blue structural color in Maratus occasus, as it does in Maratus splendens. The interior and exterior structure is evident in the micrograph of a broken plate-like scale in Figures $4 \mathrm{a}$ and $4 \mathrm{~b}$. For the MyScope Explore demonstration, an intact plate-like scale is zoomed in upon (Figure 5a). While the interior structure of the plate-like scale is not visible, the exterior parallel ridges with a periodicity of $120 \mathrm{~nm}$ are visible; students were directed to observe these during the workshop.

Students were provided with a background tutorial introducing the origin of both structural color and pigmented color, which is attributed to chemical molecules rather than nanoscale structures. Ommochromes such as xanthommatin [13] have been demonstrated to be the main pigments in spiders, in general $[15,16]$. In addition, structurally assisted absorption mechanisms have also been proposed for the brush-like scales of some Maratus species [14]. Multiple scattering between the spikes and iterative absorption can occur as light propagates through the cuticle into an absorbing layer of melanin pigment in the abdomen [14].

\section{MyScope Explore Background}

This remote learning solution made use of the existing online learning infrastructure provided by Microscopy Australia. Microscopy Australia (supported by NCRIS, the Australian Government's National Collaborative Research Infrastructure Strategy) is a national grid of equipment, instrumentation, and expertise in microscopy and microanalysis. This national grid provides 

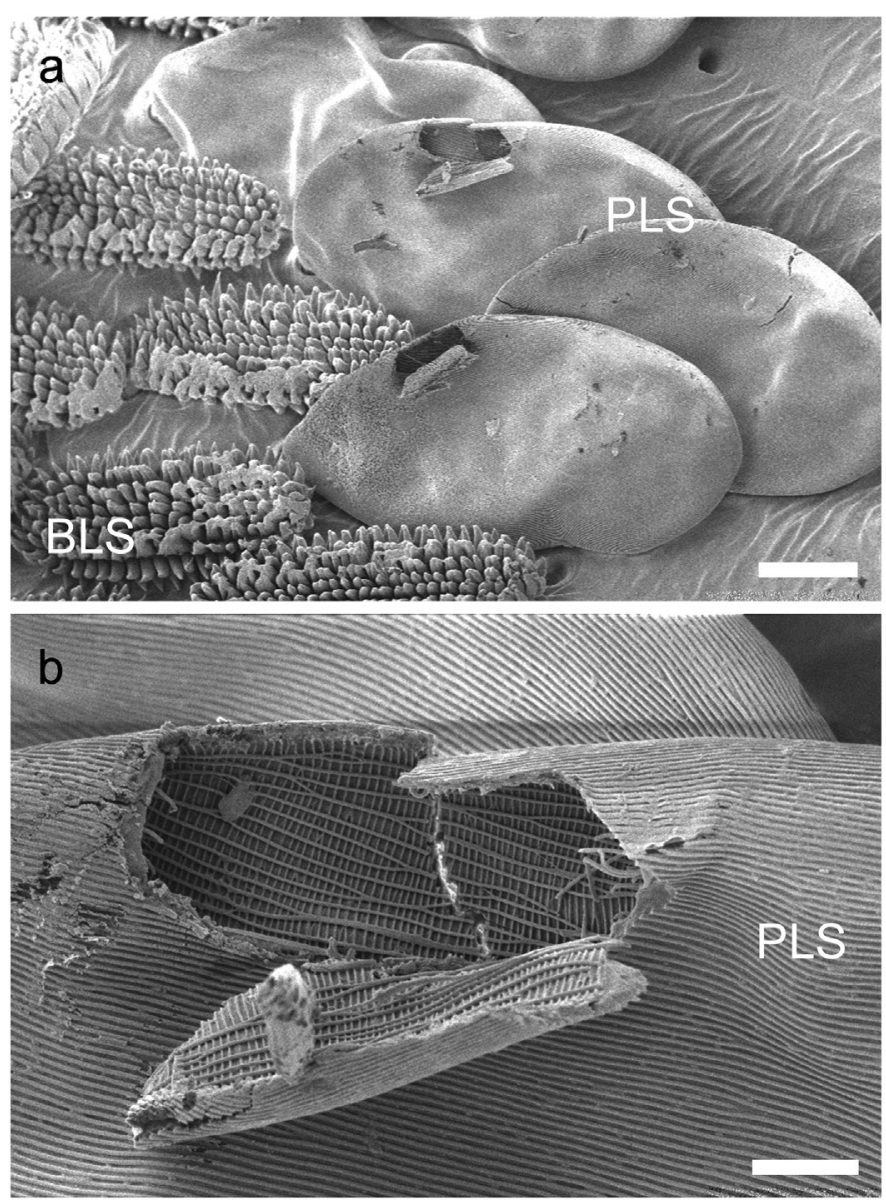

Figure 4: Scanning electron micrographs depicting (a) blue plate-like scales (PLS) (Type I) and orange brush like-scales (BLS) (Type II) of a Maratus occasus (male) specimen (scale bar=10 $\mathrm{m}$ ). (b) The internal structure of the plate-like scales of Maratus occasus that leads to the blue structural color (scale bar $=2 \mu \mathrm{m})$.

open-access nanostructural characterization capability and services, from pulsed-laser local electrode atom probe tomography and high-precision focused ion microprobes to high-resolution SEM and high-throughput cryo-transmission electron microscopy. The collaborative facility comprises a distributed network of microscopy and microanalysis core facilities across nine institutions (University of Sydney, University of Queensland, University of New South Wales, Australian National University, University of Western Australia, Flinders University, University of Adelaide, University of South Australia, Monash University). MyScope was developed by the participating universities to support the face-to-face training offered within their facilities but has evolved into one of the best-known and heavily used training tools for microscopy worldwide.

The MyScope SEM simulation is available in two differing versions:

1. A simplified simulation of an SEM instrument that explains each of the different instrument controls and what their effect on an SEM image will be (for example, evacuate the chamber to remove air molecules that will interfere with the electron beam). Students are prompted to learn the influence of various controls through this environment, by loading simulated samples from the specimen library and choosing imaging conditions. This is "MyScope Explore," which is aimed at a general and younger audience where they can both learn to use the SEM and also make discoveries by exploring over 70 stored samples.

2. A realistic SEM simulation environment that mimics an actual state-of-the-art instrument. The students can develop advanced skills through active learning activities designed to teach the impact of realistic setting changes on a sample under measurement, with a full range of controls including detector type and astigmatism correction. This is "MyScope," a professional training environment.

Here we briefly detail the programming behind the MyScope Explore graphical user interface (GUI) that makes the online interface possible. For each specimen type in the MyScope Explore library, the simulation is built using the HTML5 canvas element. First, a series of scanning electron micrographs of the specimen are embedded, each at double the magnification of the previous item in the image string (Figure 5). The canvas element is used to write three image filters: blur, contrast, and brightness. The micrographs are then drawn onto the canvas, the canvas pixels are read back, and the three image filters run
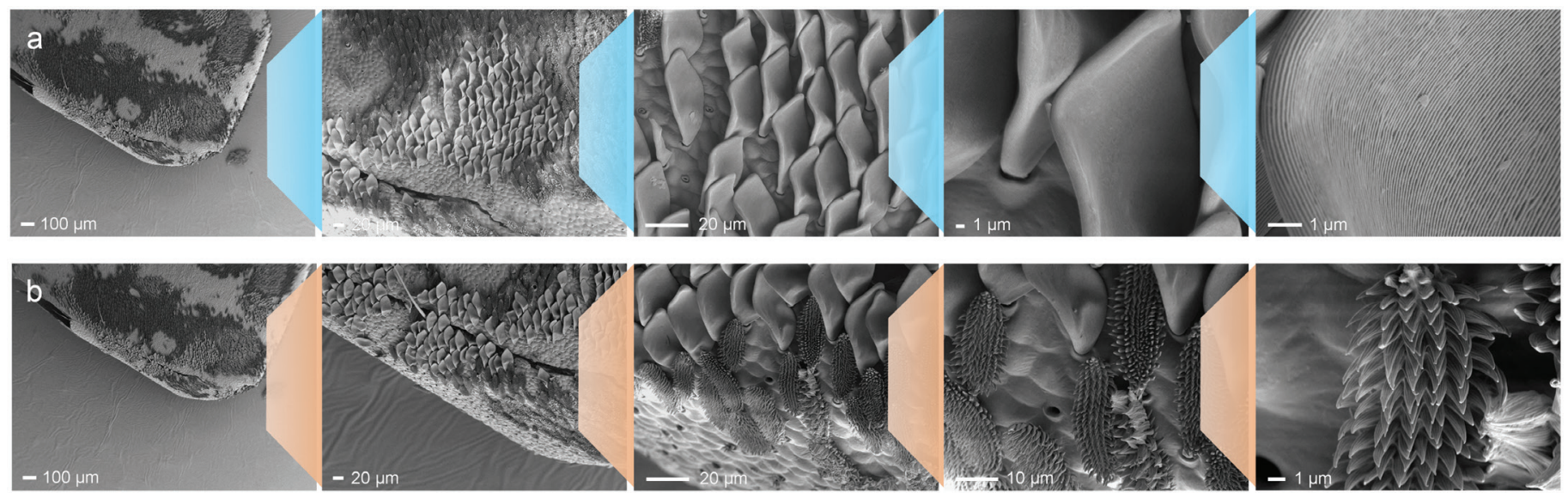

Figure 5: A subset of the scanning electron micrographs of Maratus occasus embedded into MyScope Explore under the "Arthropods" category. (a) Zoom series for color 1 (blue plate-like scales, Type I). (b) Zoom series for color 2 (orange brush-like scales, Type II). 
on them using JavaScript. The simulation includes Accelerating Voltage, Spot Size, Z Height Distance, Brightness, Contrast, Focus, and Magnification functions. The Magnification function is established by replacing the image with the next image in the sequence, the Focus function operates by applying a blur, to varying degrees, both above and below the focus point. The Accelerating Voltage, Spot Size, and Z Height Distance functions operate based on a combination, to varying degrees, of the brightness, contrast, and blur filters. Hence, as new teaching and outreach activities arise, new specimens can be programmed into the MyScope Explore platform to benefit the microscopy community, as was done for Maratus occacus specifically for the University of Newcastle 2020 workshop.

During the workshop, the students were instructed to open MyScope Explore and load the SEM simulation for both Type I and Type II scales of the Maratus occasus specimen. They were then directed to optimize the SEM image quality, firstly without prompting, then with an increasing degree of guidance as the session progressed. Students were directed to show the activity coordinator an optimized in-focus micrograph of each scale type once achieved. The students performed the following steps in order to complete the MyScope Explore simulation for each scale type:

1. Choose the sample.

2. Load the sample, and press the evacuate button.

3. Select accelerating voltage (options include 5, 10, 15, 20, and $30 \mathrm{kV}$ ).

4. Select a spot size (options include 5, 10, 15, and $20 \mathrm{~nm}$ ).

5. Select a $Z$ height distance (options include 8, 10, and $20 \mathrm{~mm})$.

6. Select HV ON.

7. Adjust brightness using the scroll bar.

8. Adjust contrast using the scroll bar.

9. Adjust focus using the scroll bar.

10. Adjust magnification using the scroll bar.

11. Save and/or print the resultant micrograph.
Note that upon loading a sample, the starting point for the focus, contrast, and brightness is randomized in the MyScope Explore software such that it differs each time the same sample is loaded. This ensures that each student must optimize these settings for each increase in magnification rather than being able to copy another student's settings.

\section{Teaching Scanning Electron Microscopy via Zoom}

The interactive live SEM session component of the program was performed at the EMX facility at the University of Newcastle, with students joining the session remotely via Zoom from a computer lab on campus with a large seating capacity. The usual 45-minute sessions were expanded to 90-minute sessions and were scheduled four times across the 3-day workshop program (Figure 6). This change ensured that all students could perform the activity in manageable class sizes of 10-12 whilst maintaining the social distancing requirements for the computer laboratories. There were two Zoom channels coming through to the students from the SEM lab. The first was a screen-share to the SEM computer, showing the SmartSEM user interface live during imaging, whilst the second was a video link of the scientist performing the measurements so that the students could see the sample loading and operation of the keyboard controls for functions including focus, magnification, etc. A standard optical microscope was also assembled in the computer laboratory to enable students to physically load a Maratus occasus specimen and view the vibrant colors, and then match the colors to the grayscale SEM images of the nanostructured scales of a duplicate specimen loaded into the SEM instrument at the EMX facility.

The scientist operating the SEM recorded micrographs of the Peacock spider specimen by asking a series of prompt questions of the student cohort watching via Zoom and recording the accuracy, timeliness, and level of cohort agreement for the answers. This practical SEM session aimed to estimate how successful the students would work as "independent" operators of the microscope. 12:00pm-1:15pm

GROUP ACTIVITIES

1:15pm-2:00pm

$2: 00 p m-2: 45 p m$

$2: 45 \mathrm{pm}-3: 30 \mathrm{pm}$

3:30pm - 4:15pm

4:15pm-5:00pm

LUNCH - Physics Grassed Courtyard Synthesis \& Spec-
troscopy (58209)

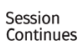
Continues
(SB209) R2R Printing/NI R2R Printing
Tour
(NIIER A Blodid Solar Blinds/ Large A (MIER Outdoor Area)

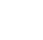




\section{Student Engagement}

Following the workshop, students were asked to comment on their experience, with the feedback from the students being very positive-an indication that student engagement was high. Comments from students included, "Experimenting with multiple imaging settings for the SEM simulation improved my understanding of the equipment's utility in real lab settings," and "Two-way communication (Zoom SEM session) was way better than a PowerPoint presentation, and the online simulation tool allowed us to have valuable personal experiences with the SEM.” In addition, it was noted that the choice of specimen was a critical feature in promoting student engagement during the remote learning activity, as Peacock (Maratus) spiders are a species of great interest due to their well-known "spider dance" and their vibrant structural colors.

\section{Conclusion}

In summary, we have developed a remote learning electron microscopy program for undergraduate university students during the COVID-19 pandemic at the University of Newcastle. In addition to learning electron microscopy via both an online simulation and an interactive live SEM session, the students learned about structural color, that is, color that originates from the precise and periodic nanostructure of a specimen. Structurally colored features require high-resolution microscopy tools to investigate and are, hence, an ideal choice for an electron microscopy workshop. The MyScope Explore online platform enabled the students to learn independently and to experience all of the steps in imaging a nanostructured sample with SEM, providing an enriching learning experience for students during a year with strict access limitations to existing scientific infrastructure.

\section{Acknowledgements}

Biological photographer Michael Doe from Project Maratus is thanked for contribution of Maratus spider specimens; Project Maratus promotes research into the iconic Australian Peacock (Maratus) spiders. We acknowledge University of Newcastle undergraduate students Isaac A. Gill and Benjamin Stanwell for their assistance in coordinating the Spring School microscopy session; Microscopy Australia staff member Susan Warner for facilitating the new content entry into MyScope Explore; and Andres Vasquez for programming in MyScope Explore. Graphic designer Nicole Weaver is thanked for contributing Figure 6 - creative design of schedule. The authors acknowledge the research facilities and expertise supported by Microscopy Australia and the University of Sydney. We further acknowledge the technical assistance provided by Sydney Microscopy and Microanalysis, a Core Research Facility of the University of Sydney. This work was performed in part at the Materials Node (Newcastle) of the Australian National Fabrication Facility (ANFF), which is a company established under the National Collaborative Research Infrastructure Strategy to provide nano- and micro-fabrication facilities for Australia's researchers. The University of Newcastle Electron Microscopy and X-ray (EMX) Unit provided access to electron microscopes.

\section{References}

[1] B Cribb et al., Microsc Microanal 17 (2011) https://doi .org/10.1017/s1431927611005228.

[2] M Apperley et al., Microsc Microanal 21 (2015) https://doi .org/10.1017/s1431927615003438.
[3] C Fuery et al., Microsc Microanal 23 (2017) https://doi .org/10.1017/s1431927617012168.

[4] NC Nicolaidis et al., J Chem Educ 97 (2020) https://doi .org/10.1021/acs.jchemed.9b00941.

[5] J Schubert and R Whyte, Peckhamia 187.1 (2019) 1-10. ISSN 1944-8120.

[6] VJ Lloyd and NJ Nadeau, Curr Opin Genet Dev 69 (2021) https://doi.org/10.1016/j.gde.2021.01.004.

[7] J Zi et al., PNAS 100 (2003) https://doi.org/10.1073/ pnas. 2133313100 .

[8] S Kariko et al., J Royal Soc Interface 15 (2018) https://doi .org/10.1098/rsif.2017.0930.

[9] BK Hsiung et al., Nat Commun 8 (2017) https://doi .org/10.1038/s41467-017-02451-x.

[10] AE Seago et al., J Royal Soc Interface 6 (2009) https://doi .org/10.1098/rsif.2008.0354.focus.

[11] F Kopnov et al., Synthetic Metals 137 (2003) https://doi .org/10.1016/S0379-6779(02)00839-1.

[12] Y Liu et al., Nat Commun 10 (2019) https://doi.org/10.1038/ s41467-019-12360-w.

[13] DG Stavenga et al., J Royal Soc Interface 13 (2016) https:// doi.org/10.1098/rsif.2016.0437.

[14] DE McCoy et al., Proc Royal Soc B Biol Sci 286 (2019) https://doi.org/10.1098/rspb.2019.0589.

[15] TC Insausti and J Casas, J Exper Biol 211 (2008) https:// doi.org/10.1242/jeb.014043.

[16] VL Seligy, Comp Biochem Physiol Part A Physiol 42 (1972) https://doi.org/10.1016/0300-9629(72)90448-3.

\section{Refurbishing or servicing an electron microscope? Scope us out for your ion pump needs.}

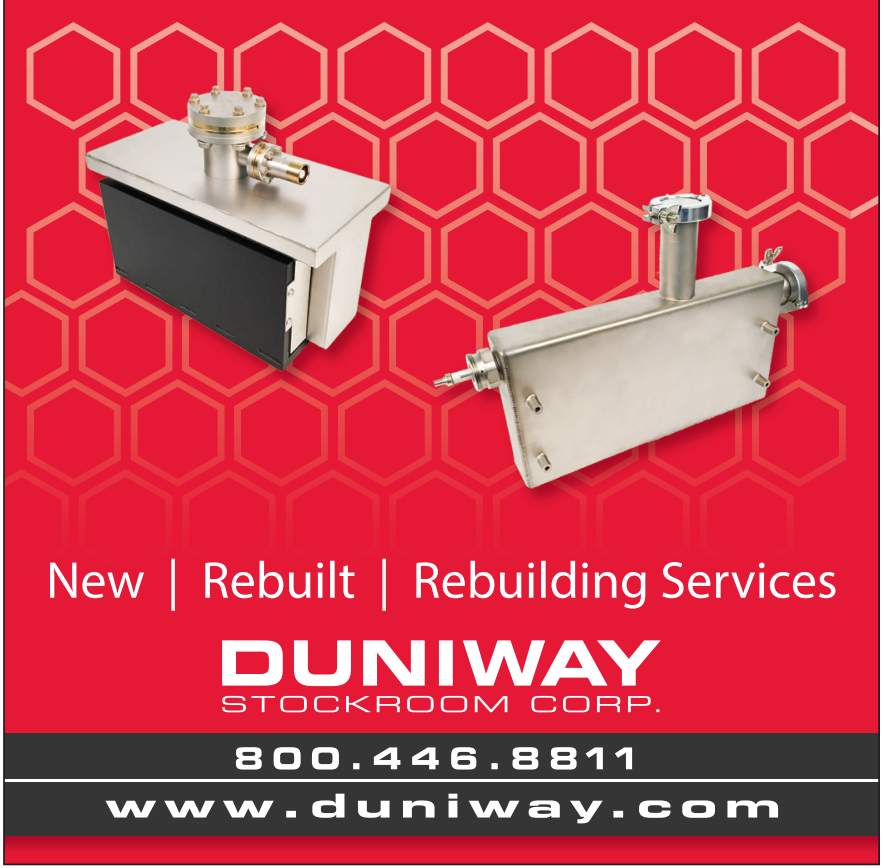




\section{First $\gg \gg\rangle$}

\section{HIGH THROUGHPUT SEM System}

\section{Navigator-10}

(6) 10 times faster than

(1) Direct electron detection technology and Dual channel imaging (SE\&BSE)

Intelligent atlas and cross-scale material characterization

(बig data intelligent analysis and Al computing
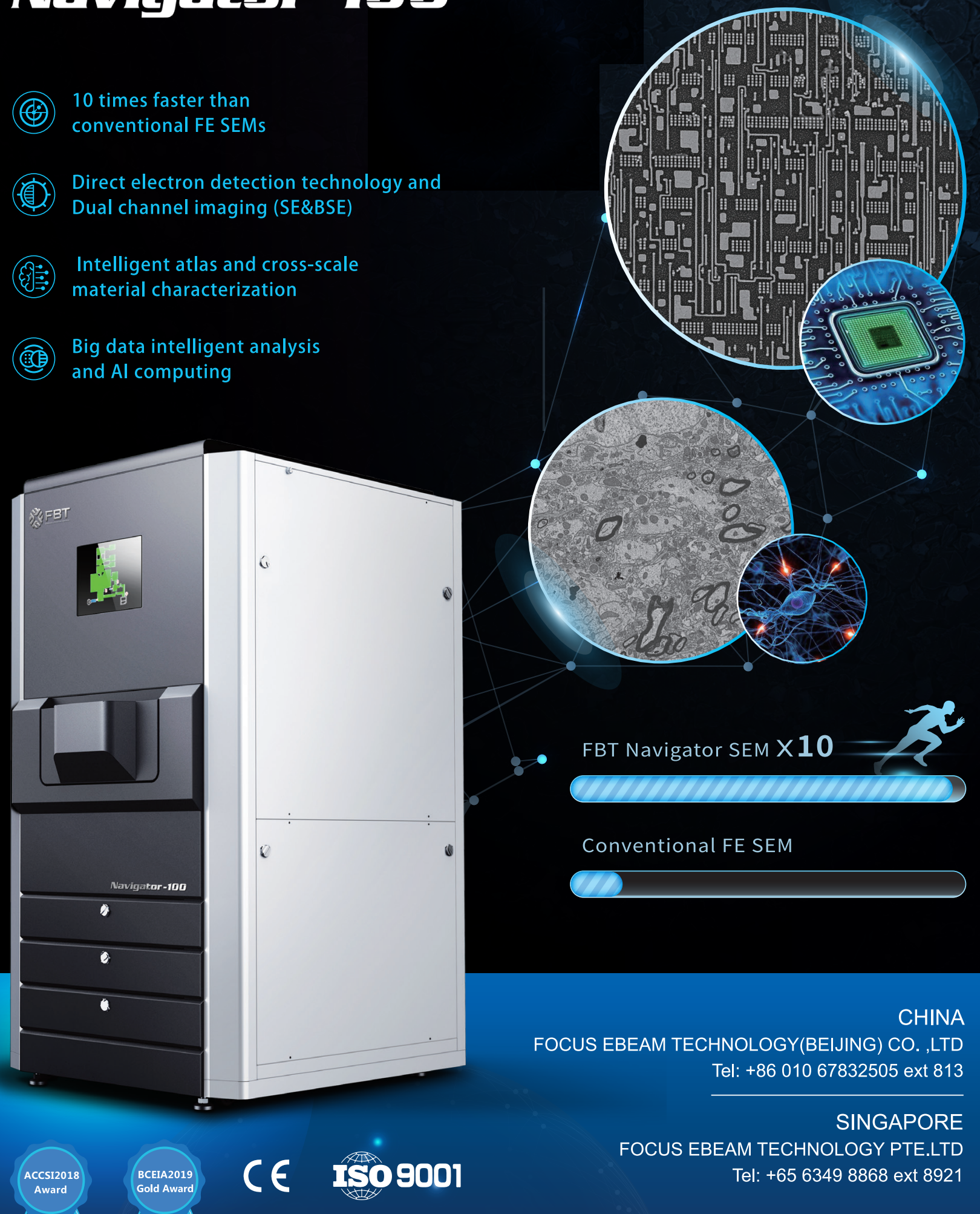

CHINA

FOCUS EBEAM TECHNOLOGY(BEIJING) CO. ,LTD

Tel: +86 01067832505 ext 813

SINGAPORE

FOCUS EBEAM TECHNOLOGY PTE.LTD

Tel: +65 63498868 ext 8921 